\title{
QUANTUM OPTIMAL TRANSPORT IS CHEAPER
}

EMANUELE CAGLIOTI, FRANÇOIS GOLSE, AND THIERRY PAUL

\begin{abstract}
AвsтRact. We compare bipartite (Euclidean) matching problems in classical and quantum mechanics. The quantum case is defined after a quantum version of the Wasserstein distance introduced in [7]. We show that the optimal quantum cost can be cheaper than the classical one. We treat in detail the case of two particles: the case of equal mass provides equal quantum and classical costs, while we exhibit examples of different masses for which the quantum cost is actually strictly cheaper.
\end{abstract}

\section{Contents}

1. Introduction

2. The equal mass case

3. The unequal mass case

4. Concluding remarks on quantum optimal transport References

\section{INTRODUCTION}

The paradigm of modern optimal transport theory uses extensively the 2-Wasserstein distance between two probability measures $\mu, \nu$ on $\mathbf{R}^{n}$, defined as

$$
W_{2}(\mu, \nu)^{2}:=\inf _{\Pi \text { coupling of } \mu \text { and } \nu} \int|x-y|^{2} \Pi(d x, d y) .
$$

We have called coupling (or transport plan) of the two probabilities $\mu$ and $\nu$ any probability measure $\Pi(d x, d y)$ on $\mathbf{R}^{n} \times \mathbf{R}^{n}$ whose marginals on the first and the second factors are $\mu$ and $\nu$ resp., i.e.

$$
\begin{aligned}
& \int_{\mathbf{R}^{n} \times \mathbf{R}^{n}} a(x) \Pi(d x, d y)=\int_{\mathbf{R}^{n}} a(x) \mu(d x), \\
& \int_{\mathbf{R}^{n} \times \mathbf{R}^{n}} b(y) \Pi(d x, d y)=\int_{\mathbf{R}^{n}} b(y) \nu(d y)
\end{aligned}
$$

for all test (i.e. continuous and bounded) functions $a$ and $b$.

Restricting the definition of $W_{2}$ to couplings of the form

$$
\Pi=\delta(y-T(x)) \mu(d x)
$$

where $T$ is a transformation of $\mathbf{R}^{n}$ such that $\nu$ is the image $T_{\#} \mu$ of $\mu$ by $T$, one sees that:

$$
M(\mu, \nu)^{2}:=\inf _{T_{\#} \mu=\nu} \int_{\mathbf{R}^{n}}(x-T(x))^{2} \mu(d x) \geq W_{2}(\mu, \nu)^{2} .
$$


The converse inequality is due to Knott, Smith and Brenier: under certain restrictions on the regularity of $\mu$ and $\nu$, any optimal coupling for the minimization problem defined by (11) is of the form (3) for some transport map $T$, so that the inequality in (4) is an equality (see e.g. [3] Section 1 for some details and Theorem 2.12 in [14] for an extensive study).

Associated to $W_{2}$ is the bipartite matching problem which can be described as follows. Let us consider $M$ material points on the real line $\left\{x_{i}\right\}_{i=1, \ldots, M}$ with $x_{i}<x_{i+1}$, and with masses $\left\{m_{i}\right\}_{i=1, \ldots, M}$, and on the other hand $N$ points $\left\{y_{i}\right\}_{i=1, \ldots, N}$ with $y_{j}<y_{j+1}$, and with masses $\left\{n_{i}\right\}_{i=1, \ldots, N}$. We normalize the total mass as follows:

$$
\sum_{i=1}^{M} m_{i}=\sum_{j=1}^{N} n_{j}=1 .
$$

The bipartite problem consists in finding a coupling matrix $\left.\left(p_{i, j}\right)\right|_{i=1, \ldots, N, j=1, \ldots M}$ satisfying

$$
\sum_{j=1}^{N} p_{i, j}=m_{i}, \quad \sum_{i=1}^{M} p_{i, j}=n_{j}, \quad p_{i, j} \geq 0 \text { for each } i, j
$$

which minimizes the quantity $\sum_{i, j} p_{i, j}\left|x_{i}-y_{j}\right|^{2}$.

That is to say, we define the optimal transport cost as

$$
\begin{aligned}
& C_{c}:=\inf _{p_{i, j} \geq 0} \sum_{i, j} p_{i, j}\left|x_{i}-y_{j}\right|^{2} . \\
& \sum_{j=1}^{N} p_{i, j}=m_{i}, \sum_{i=1}^{M} p_{i, j}=n_{j}
\end{aligned}
$$

It is natural to associate to the sets $\left\{x_{i}\right\}_{i=1, \ldots, M}$ and $\left\{m_{i}\right\}_{i=1, \ldots, M}$, and to the sets $\left\{y_{i}\right\}_{i=1, \ldots, N}$ and $\left\{n_{i}\right\}_{i=1, \ldots, N}$ the following discrete probability measures

$$
\mu:=\sum_{i=1}^{M} m_{i} \delta_{x_{i}}, \quad \nu:=\sum_{j=1}^{N} n_{j} \delta_{y_{j}} .
$$

It is easy to see that any optimal coupling of $\mu, \nu$ for $W_{2}$ takes the form

$$
\Pi=\sum_{i, j} p_{i, j} \delta_{x_{i}} \otimes \delta_{y_{j}}, \quad \text { i.e. } \quad \Pi(x, y)=\sum_{i, j} p_{i, j} \delta\left(x-x_{i}\right) \delta\left(y-y_{j}\right),
$$

so that

$$
C_{c}=W_{2}(\mu, \nu)^{2} .
$$

A general review of the bipartite problem is out of the scope of the present paper, and the reader is referred to the seminal work [11], the thesis [13] which contains an extensive bibliography, and [2] for a lucid presentation of the mathematical theory pertaining to this problem. Let us describe the simplest case $M=N=2$.

In the case of equal masses, that is $m_{1}=m_{2}=n_{1}=n_{2}=\frac{1}{2}$, the optimal coupling is shown to be diagonal, in the sense that the mass $\frac{1}{2}$ is transported from the point $x_{1}$ to 


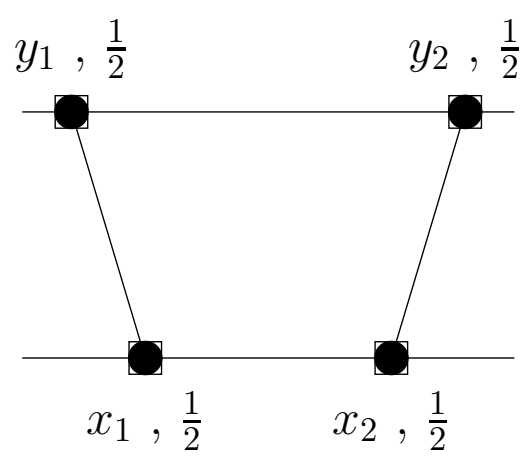

Figure 1. Equal masses

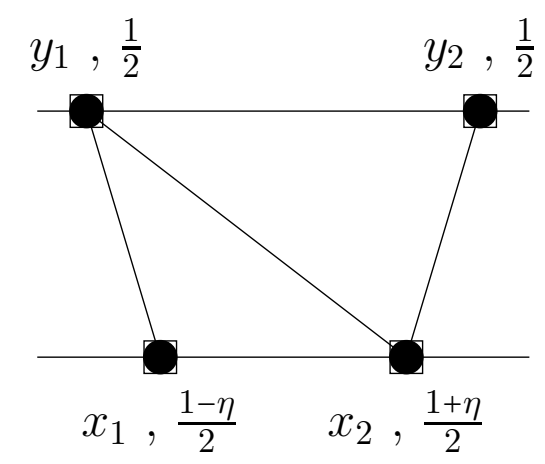

FIGURE 2. Different masses

the point $y_{1}$, and likewise for $x_{2}$ and $y_{2}$. Thus

$$
\Pi_{o p}=\frac{1}{2} \delta_{x_{1}} \otimes \delta_{y_{1}}+\frac{1}{2} \delta_{x_{2}} \otimes \delta_{y_{2}}
$$

or equivalently

$$
\Pi_{o p}(x, y)=\frac{1}{2} \delta\left(x-x_{1}\right) \delta\left(y-y_{1}\right)+\frac{1}{2} \delta\left(x-x_{2}\right) \delta\left(y-y_{2}\right)
$$

and therefore

$$
C_{c}=\frac{1}{2}\left(x_{1}-y_{1}\right)^{2}+\frac{1}{2}\left(x_{2}-y_{2}\right)^{2}
$$

In the case of unequal masses, let us consider the example where $m_{1}=\frac{1-\eta}{2}$ and $m_{2}=\frac{1+\eta}{2}$ for some $0<\eta<1$, while $n_{1}=n_{2}=\frac{1}{2}$. In this case, one shows that the optimal transport moves the mass $\frac{1}{2}$ from $x_{2}$ to $y_{2}$, moves the remaining amount of the mass at $x_{2}$, i.e. $\frac{\eta}{2}$, from $x_{2}$ to $y_{1}$, and finally moves the mass $\frac{1-\eta}{2}$ from $x_{1}$ and $y_{1}$. Therefore, the optimal coupling in this case is

$$
\Pi_{o p}(x, y)=\frac{1-\eta}{2} \delta\left(x-x_{1}\right) \delta\left(y-y_{1}\right)+\frac{\eta}{2} \delta\left(x-x_{2}\right) \delta\left(y-y_{1}\right)+\frac{1}{2} \delta\left(x-x_{2}\right) \delta\left(y-y_{2}\right),
$$

so that

$$
C_{c}=\frac{1-\eta}{2}\left(x_{1}-y_{1}\right)^{2}+\frac{\eta}{2}\left(x_{2}-y_{1}\right)^{2}+\frac{1}{2}\left(x_{2}-y_{2}\right)^{2} .
$$


A quantum analogue to the Wasserstein distance has been recently introduced in [7] according to the following general fact.

When passing from classical to quantum mechanics,

1. functions on phase-space should be replaced by operators on the Hilbert space of square integrable functions on the underlying configuration space, and

2. integration (over phase space) of classical functions should be replaced by the trace of the corresponding operators. Moreover,

3. coordinates $q$ of the configuration space should be replaced by the multiplication operator $\hat{q}$ by the $q$ variable, while momentum coordinates $p$ should be replaced by the operator $\hat{p}=-i \hbar \nabla$.

These considerations are in full accordance with the definition of quantum density matrices as self-adjoint positive operators of trace 1 on $\mathfrak{H}:=L^{2}\left(\mathbf{R}^{d}\right)$. They are also consistent with the definition of couplings $Q$ of two density matrices $R$ and $S$ as density matrices on $\mathfrak{H} \otimes \mathfrak{H}$ (identified with $L^{2}\left(\mathbf{R}^{2 d}\right)$ ) whose marginals (defined consistently again as partial traces on the two factors of $\mathfrak{H} \otimes \mathfrak{H})$ are equal to $R$ and $S$. In other words

$$
\operatorname{trace}_{\mathfrak{H} \otimes \mathfrak{H}}\left(\left(A \otimes I_{\mathfrak{H}}\right) Q\right)=\operatorname{trace}_{\mathfrak{H}}(A R), \quad \operatorname{trace}_{\mathfrak{H} \otimes \mathfrak{H}}\left(\left(I_{\mathfrak{H}} \otimes B\right) Q\right)=\operatorname{trace}_{\mathfrak{H}}(B S)
$$

for all bounded operators $A, B$ on $\mathfrak{H}$, by analogy with (2).

Moreover they lead naturally to the following definition of the analogue of the Wasserstein distance between two quantum densities $R$ and $S$. Consistently with (1) expressed on the phase-space $\mathbf{R}^{2 d}$, therefore with $n=2 d$, we define $M K_{2} \geq 0$ by

$$
M K_{2}(R, S)^{2}:=\inf _{Q \text { coupling of } R \text { and } S} \operatorname{trace}(C Q),
$$

with

$$
C:=(\hat{p} \otimes \mathrm{I}-I \otimes \hat{p})^{2}+(\hat{q} \otimes I-I \otimes \hat{q})^{2}-2 d \hbar .
$$

In other words, expressed as an operator on $L^{2}\left(\mathbf{R}^{d}, d x\right) \otimes L^{2}\left(\mathbf{R}^{d}, d y\right)$,

$$
C=(x-y)^{2}-\hbar^{2}\left(\nabla_{x}-\nabla_{y}\right)^{2}-2 d \hbar=-4 \hbar^{2} \nabla_{x-y}^{2}+(x-y)^{2}-2 d \hbar .
$$

The operator $\frac{1}{2}(C+2 d \hbar)$ is a quantum harmonic oscillator in the variable $(x-y) / \sqrt{2}$, and this implies in particular that $C=C^{*} \geq 0$.

The quantity $M K_{2}$ is not a distance as shown in [7] p. 171. Nevertheless, it was established in [7] the two following links between $M K_{2}$ and $W_{2}{ }_{2}^{1}$. First, for any pair of density matrices $R$ and $S$, the Husimi functions $\widetilde{W}[R]$ and $\widetilde{W}[S]$ of $R$ and $S$ satisfy

$$
W_{2}(\widetilde{W}[R], \widetilde{W}[S])^{2} \leq M K_{2}(R, S)^{2}+4 d \hbar .
$$

On the other hand, if $R$ and $S$ are Töplitz operators of symbols $\mu$ and $\nu$,

$$
M K_{2}(R, S)^{2} \leq W_{2}(\mu, \nu)^{2} .
$$

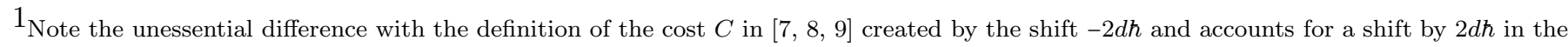
two next formulas.
} 
Let us recall that a Töplitz operator $T$ (or positive quantization, or anti-Wick ordering quantization) of symbol a probability measure $\tau$ is ${ }^{2}$

$$
T:=\int_{\mathbf{R}^{2 d}}|q, p\rangle\langle q, p| \tau(d q, d p),
$$

where $|q, p\rangle$ is a coherent state at point $(q, p)$ i.e.

$$
\langle x \mid q, p\rangle:=(\pi \hbar)^{-d / 4} e^{-(x-q)^{2} / 2 \hbar} e^{i p x / \hbar} .
$$

We also recall the definition of the Husimi function of a density matrix $R$ :

$$
\widetilde{W}[R](q, p):=(2 \pi \hbar)^{-d}\langle q, p|R| q, p\rangle .
$$

The functional $M K_{2}^{2}$ (more precisely $M K_{2}^{2}+2 d \hbar$ with the definition chosen in the present paper) has been systematically used and extended in [7, 8, 9] in order to study various problems, such as the validity of the mean-field limit uniformly in $\hbar$, the semiclassical approximation of quantum dynamics, and the problem of metrizing of the set of quantum densities in the semiclassical regime.

Given the importance of optimal transport in the field of statistics in the problem of comparing probability measures, there have been various attempts at defining analogues of the Wasserstein, or Monge-Kantorovich distances in the quantum setting. For instance, the reference [16] proposed to consider the original Monge distance (also called the Kantorovich-Rubinstein distance, or the Wasserstein distance of exponent 1) between the Husimi transforms of the density operator. However, propagating this distance with the usual quantum dynamics may not be easy, because the dynamics of the Husimi transform of a density operator by the von Neumann equation is quite involved [1]. A big advantage of the quantity $M K_{2}$ introduced in [7] is that it is directly defined in terms of density operators, and therefore easily propagated by the usual quantum dynamics, including $N$-body quantum dynamics, for which it has been defined originally. Besides the quantity $M K_{2}$ appeared in [7], other quantum analogues of the Wasserstein distance of exponent 2 have been proposed by several other authors. For instance a quantum analogue of the Benamou-Brenier formula (see Theorem 8.1 in chapter 8 of [14]) for the classical Wasserstein distance of exponent 2 is studied in detail in [4, 5], and this idea has been used to obtain a quantum equivalent of the so-called HWI inequality [12]. More recently, other propositions for generalizing Wasserstein distances to the quantum setting have emerged, such as [10] (which seems essentially focussed on pure states) or [6], which is very close to our definition of $M K_{2}$, except that the set of couplings used in the minimization is different.

The quantum bipartite problem can be therefore stated as follows, in close analogy with the classical picture introduced earlier.

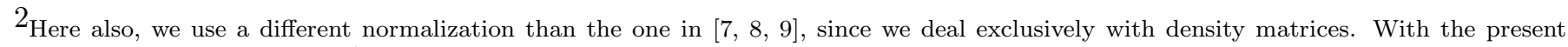
normalization, one has trace $T=\int_{\mathbf{R}^{2 d}} \tau(d q, d p)$.
} 
One considers two density matrices built in terms of the positions and masses already used for the classical bipartite problem, in the following way

$$
R=\sum_{i=1}^{M} m_{i}\left|x_{i}, 0\right\rangle\left\langle x_{i}, 0\left|, \quad S=\sum_{j=1}^{N} n_{j}\right| y_{j}, 0\right\rangle\left\langle y_{j}, 0\right| .
$$

Indeed, it is natural to associate coherent states to material points, as they saturate the Heisenberg uncertainty inequalities. Moreover, one sees that $R$ and $S$ are precisely the Töplitz operators of symbols $\mu$ and $\nu$ respectively.

The quantum bipartite problem consists then in finding an optimal coupling of $R$ and $S$ for $M K_{2}(R, S)$ and the optimal quantum cost defined as

$$
C_{q}:=M K_{2}(R, S) \text {. }
$$

Since $R$ and $S$ are Töplitz operators, we know from (5) that

$$
C_{q} \leq C_{c} \text {. }
$$

The question we address in this paper is whether there exist pairs of density matrices for which

$$
C_{q}<C_{c}
$$

In other words, we address the question of whether quantum optimal transportation can be cheaper than its classical analogue.

We shall study the two cases introduced at the beginning of this section and described in Figures 1 and 2. For the sake of simplicity, we shall take $x_{1}=-x_{2}=-a, y_{1}=-y_{2}=-b$, with $a<b$ in the equal mass case, and $a=b$ in the unequal mass case.

In the equal mass case, studied in Section 2, both classical and quantum transport are achieved without splitting mass for each particle: the two costs are shown to be equal (see (13)), and an optimal quantum coupling is the Töplitz quantization to the optimal classical coupling.

In Section 3 we study the case of different masses and construct a family of examples for which the optimal quantum cost is strictly cheaper than the classical one (see (21)).

In addition, we show in Section 4 that an optimal quantum coupling is not always the Töplitz quantization of a classical coupling. This can be rephrased by saying that an optimal quantum transport can be different from the natural quantization of any underlying classical transport. In fact, in the unequal mass case treated in this paper, no quantum optimal transport corresponds to a classical transport, optimal or not: they all involve strictly quantum effects.

\section{The EQUAL MASS CASE}

For $a, b>0$ we will transport a superposition of two density matrices which are pure states associated to two coherent states of null momenta localized at $+a$ and $-a$ towards a similar density matrix associated to the points $( \pm b, 0)$ in phase space. In 
other words, we consider the coherent states denoted $|c\rangle$ for simplicity (instead of $|c, 0\rangle$, i.e. $\left.\langle x \mid c\rangle:=(\pi \hbar)^{-1 / 4} e^{-(x-c)^{2} / 2 \hbar}\right)$ and consider the two density matrices

$$
R:=\frac{1}{2}(|a\rangle\langle a|+|-a\rangle\langle-a|), \quad S:=\frac{1}{2}(|b\rangle\langle b|+|-b\rangle\langle-b|) .
$$

Define

$$
\lambda:=\langle a \mid-a\rangle=e^{-a^{2} / \hbar}, \quad \mu:=\langle b \mid-b\rangle=e^{-b^{2} / \hbar},
$$

and consider the two pairs of orthogonal vectors

$$
\phi_{ \pm}:=\frac{|a\rangle \pm|-a\rangle}{\sqrt{2(1 \pm \lambda)}}, \quad \psi_{ \pm}:=\frac{|b\rangle \pm|-b\rangle}{\sqrt{2(1 \pm \mu)}} .
$$

Hence

$$
R=\alpha_{+}\left|\phi_{+}\right\rangle\left\langle\phi_{+}\left|+\alpha_{-}\right| \phi_{-}\right\rangle\left\langle\phi_{-}\left|, \quad S=\beta_{+}\right| \psi_{+}\right\rangle\left\langle\psi_{+}\left|+\beta_{-}\right| \psi_{-}\right\rangle\left\langle\psi_{-}\right|
$$

with

$$
\alpha_{+}:=\frac{1}{2}(1+\lambda), \quad \alpha_{-}=\frac{1}{2}(1-\lambda), \quad \beta_{+}:=\frac{1}{2}(1+\mu), \quad \beta_{-}=\frac{1}{2}(1-\mu) .
$$

In the whole present paper, we will only use couplings of $R$ and $S$ that act from the four-dimensional linear span of $\phi_{ \pm} \otimes \psi_{ \pm}$to itself. Therefore, in order to compute trace $(C Q)$ for such couplings, we need to project the cost operator $C$ on the basis $\left\{\phi_{+} \otimes \psi_{+}, \phi_{+} \otimes \psi_{-}, \phi_{-} \otimes \psi_{+}, \phi_{-} \otimes \psi_{-}\right\}$. This is a tedious but straightforward computation which results in the following $4 \times 4$ matrix:

$$
C=\left(\begin{array}{cccc}
\mathcal{A} & 0 & 0 & \gamma \\
0 & \mathcal{B} & \delta & 0 \\
0 & \delta & \mathcal{C} & 0 \\
\gamma & 0 & 0 & \mathcal{D}
\end{array}\right)
$$

where

$$
\begin{aligned}
& \mathcal{A}=a^{2} \frac{1-\lambda}{1+\lambda}+b^{2} \frac{1-\mu}{1+\mu}, \quad \mathcal{B}=a^{2} \frac{1-\lambda}{1+\lambda}+b^{2} \frac{1+\mu}{1-\mu}, \quad \gamma=-\frac{2 a b(1-\lambda \mu)}{\sqrt{\left(1-\lambda^{2}\right)\left(1-\mu^{2}\right)}}, \\
& \mathcal{C}=a^{2} \frac{1+\lambda}{1-\lambda}+b^{2} \frac{1-\mu}{1+\mu}, \quad \mathcal{D}=a^{2} \frac{1+\lambda}{1-\lambda}+b^{2} \frac{1+\mu}{1-\mu}, \quad \delta=-\frac{2 a b(1+\lambda \mu)}{\sqrt{\left(1-\lambda^{2}\right)\left(1-\mu^{2}\right)}} .
\end{aligned}
$$

As a warm up in order to find an ansatz for the general case, let us first neglect the contributions of $\lambda, \mu$, exponentially small in the Planck constant. In this case $\alpha_{ \pm}=\beta_{ \pm}=\frac{1}{2}$, and the cost is equal to

$$
C_{0}=\left(\begin{array}{cccc}
a^{2}+b^{2} & 0 & 0 & -2 a b \\
0 & a^{2}+b^{2} & -2 a b & 0 \\
0 & -2 a b & a^{2}+b^{2} & 0 \\
-2 a b & 0 & 0 & a^{2}+b^{2}
\end{array}\right)
$$


On the other hand, one has

$$
Q_{0}:=\frac{1}{4}\left(\begin{array}{cccc}
1 & 0 & 0 & 1 \\
0 & 1 & 1 & 0 \\
0 & 1 & 1 & 0 \\
1 & 0 & 0 & 1
\end{array}\right) \geq 0
$$

since the spectrum of $Q_{0}$ is easily shown to be $\left\{0, \frac{1}{2}\right\}$ by using the elementary formula

$$
\operatorname{det}\left(\begin{array}{cccc}
\bar{a} & 0 & 0 & \gamma \\
0 & \bar{b} & \delta & 0 \\
0 & \delta & \bar{c} & 0 \\
\gamma & 0 & 0 & \bar{d}
\end{array}\right)=\left(\bar{a} \bar{d}-\gamma^{2}\right)\left(\bar{b} \bar{c}-\delta^{2}\right) \text { for all } \bar{a}, \bar{b}, \bar{c}, \bar{d}, \gamma, \delta
$$

Moreover, one easily checks that trace $_{2} Q_{0}=R$ and trace $Q_{0}=S$ so that $Q_{0}$ is a coupling of $R$ and $S$.

Another easy computation shows that

$$
\operatorname{trace}\left(C Q_{0}\right)=(a-b)^{2} \text {. }
$$

Therefore

$$
M K_{2}(R, S)^{2} \leq(a-b)^{2}=W_{2}\left(\frac{1}{2}\left(\delta_{-a}+\delta_{a}\right), \frac{1}{2}\left(\delta_{-b}+\delta_{b}\right)\right)^{2} .
$$

For the "true" case $\lambda, \mu \neq 0$, we make the following ansatz on the coupling $Q$

$$
Q=Q_{0}+\frac{1}{4}\left(\begin{array}{cccc}
p+\lambda+\mu & 0 & 0 & u \\
0 & -p+\lambda-\mu & v & 0 \\
0 & v & -p-\lambda+\mu & 0 \\
u & 0 & 0 & p-\lambda-\mu
\end{array}\right), p, u, v \in \mathbf{R} .
$$

Straightforward computations show that

$\operatorname{trace} Q=\operatorname{trace} Q_{0}=1, \quad \operatorname{trace}_{2} Q=\operatorname{trace}_{2} Q_{0}=R, \quad \operatorname{trace}_{1} Q=\operatorname{trace}_{1} Q_{0}=S$.

Using again (8) shows that

$$
Q \geq 0 \Longleftrightarrow-1+\sqrt{(\lambda+\mu)^{2}+(1+u)^{2}} \leq p \leq 1-\sqrt{(\lambda-\mu)^{2}+(1+v)^{2}} .
$$

Therefore, assuming that $p, u, v$ satisfy this constaint, $Q$ is a coupling of $R$ and $S$.

Denoting $U:=1+u$ and $V:=1+v$, we compute $W:=\operatorname{trace}(C Q)$ by using (7):

$$
\begin{aligned}
4 W= & 2 \gamma U+2 \delta V+p(\mathcal{A}-\mathcal{B}-\mathcal{C}+\mathcal{D})+\mathcal{A}+\mathcal{B}+\mathcal{C}+\mathcal{D} \\
& +(\lambda+\mu)(\mathcal{A}-\mathcal{D})+(\lambda-\mu)(\mathcal{B}-\mathcal{C}) \\
= & 2 \gamma U+2 \delta V+p(\mathcal{A}-\mathcal{B}-\mathcal{C}+\mathcal{D})+W^{\prime}=2 \gamma U+2 \delta V+W^{\prime}
\end{aligned}
$$

with

$$
\begin{aligned}
W^{\prime} & :=\mathcal{A}+\mathcal{B}+\mathcal{C}+\mathcal{D}+\lambda(\mathcal{A}+\mathcal{B}-\mathcal{C}-\mathcal{D})+\mu(\mathcal{A}-\mathcal{B}+\mathcal{C}-\mathcal{D}) \\
& =4\left(a^{2} \frac{1+\lambda^{2}}{1-\lambda^{2}}+b^{2} \frac{1+\mu^{2}}{1-\mu^{2}}\right)-8 a^{2} \frac{\lambda^{2}}{1-\lambda^{2}}-8 b^{2} \frac{\mu^{2}}{1-\mu^{2}} \\
& =4\left(a^{2}+b^{2}\right)
\end{aligned}
$$


Since $W$ is linear in $U, V$, we minimize $\gamma U+\delta V$ by taking

$$
U=\sqrt{(p+1)^{2}-(\lambda+\mu)^{2}} \quad \text { and } \quad V=\sqrt{(p-1)^{2}-(\lambda-\mu)^{2}},
$$

and, since $\delta \leq \gamma$, we conclude that

$$
4 W=2 T+W^{\prime}
$$

where

$$
\begin{gathered}
T=-\max _{-1+\lambda-\mu \leq p \leq 1-(\lambda-\mu)}\left(-\gamma \sqrt{(p+1)^{2}-(\lambda+\mu)^{2}}-\delta \sqrt{(p-1)^{2}-(\lambda-\mu)^{2}}\right) \\
=\frac{-2 a b}{\sqrt{\left(1-\lambda^{2}\right)\left(1-\mu^{2}\right)}}-\max _{-1+\lambda-\mu \leq p \leq 1-(\lambda-\mu)}\left((1-\lambda \mu) \sqrt{(p+1)^{2}-(\lambda+\mu)^{2}}\right. \\
\left.+(1+\lambda \mu) \sqrt{(p-1)^{2}-(\lambda-\mu)^{2}}\right) .
\end{gathered}
$$

One can check that the max is attained for $p=\lambda \mu \rightarrow 0$ as $\hbar \rightarrow 0$, and that

$$
T=-\frac{4 a b}{\sqrt{\left(1-\lambda^{2}\right)\left(1-\mu^{2}\right)}} \sqrt{1+\lambda^{2} \mu^{2}-\lambda^{2}-\mu^{2}}=-4 a b .
$$

Eventually, we arrive at the same result as in the semiclassical regime $\lambda=\mu=0$, viz.

$$
M K_{2}(R, S)^{2} \leq(a-b)^{2} .
$$

Since $R$ and $S$ are Töplitz operator, the inequality (9) was already known by using (5). Nevertheless we gave this explicit computation as we believe the result to be valid for more general density matrices.

In order to get a lower bound for $M K_{2}(R, S)$, we shall use a dual version of the definition of $M K_{2}$, proved in [3], that is a quantum version of the Kantorovitch duality theorem for $W_{2}$ (see [14, 15]):

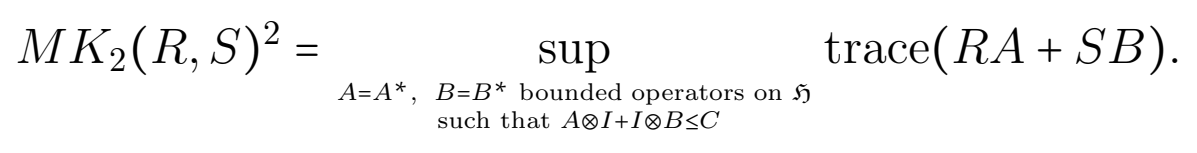

We make the following diagonal ansatz on $A$ and $B$ :

$$
A=\left(\begin{array}{cc}
\alpha_{1} & 0 \\
0 & \alpha_{2}
\end{array}\right) \quad B=\left(\begin{array}{cc}
\beta_{1} & 0 \\
0 & \beta_{2}
\end{array}\right)
$$

so that

$$
A \otimes I=\left(\begin{array}{cccc}
\alpha_{1} & 0 & 0 & 0 \\
0 & \alpha_{1} & 0 & 0 \\
0 & 0 & \alpha_{2} & 0 \\
0 & 0 & 0 & \alpha_{2}
\end{array}\right) \quad \text { and } \quad I \otimes B=\left(\begin{array}{cccc}
\beta_{1} & 0 & 0 & 0 \\
0 & \beta_{2} & 0 & 0 \\
0 & 0 & \beta_{1} & 0 \\
0 & 0 & 0 & \beta_{2}
\end{array}\right)
$$


Hence

and, according to (7),

$$
A \otimes I+I \otimes B-C:=\left(\begin{array}{cccc}
\bar{a} & 0 & 0 & -\gamma \\
0 & \bar{b} & -\delta & 0 \\
0 & -\delta & \bar{c} & 0 \\
-\gamma & 0 & 0 & \bar{d}
\end{array}\right),
$$

$$
\bar{a}=\alpha_{1}+\beta_{1}-\mathcal{A}, \quad \bar{b}=\alpha_{1}+\beta_{2}-\mathcal{B}, \quad \bar{c}=\alpha_{2}+\beta_{1}-\mathcal{C}, \quad \bar{d}=\alpha_{2}+\beta_{2}-\mathcal{D} .
$$

Notice that

$$
\bar{a}+\bar{d}=\bar{b}+\bar{c}
$$

Using (8) to compute the characteristic polynomial of $A \otimes I+I \otimes B-C$, we find that

$$
A \otimes I+I \otimes B \leq C \Longleftrightarrow \bar{a}+\bar{d} \leq-\sqrt{(\bar{a}-\bar{d})^{2}+4 \gamma^{2}} \text { and } \bar{b}+\bar{c} \leq-\sqrt{(\bar{b}-\bar{c})^{2}+4 \delta^{2}} .
$$

Moreover,

$$
\begin{aligned}
\operatorname{trace}(A R+B S) & =\frac{1}{2}\left(\alpha_{1}+\alpha_{2}+\beta_{1}+\beta_{2}\right)+\frac{\lambda}{2}\left(\alpha_{1}-\alpha_{2}\right)+\frac{\mu}{2}\left(\beta_{1}-\beta_{2}\right) \\
& =\frac{1}{4}(\bar{a}+\bar{b}+\bar{c}+\bar{d})+\frac{1}{4}(\bar{a}+\bar{b}-\bar{c}-\bar{d}) \lambda \\
& +\frac{1}{4}(\bar{a}-\bar{b}+\bar{c}-\bar{d}) \mu+a^{2}+b^{2} .
\end{aligned}
$$

Let us denote

$$
x:=\bar{a}+\bar{d}=\bar{b}+\bar{c},
$$

so that

$$
\operatorname{trace}(A R+B S)=\frac{1}{2} x+\frac{1}{4}(\lambda+\mu)(\bar{a}-\bar{d})+\frac{1}{4}(\lambda-\mu)(\bar{b}-\bar{c})+a^{2}+b^{2} .
$$

The constraints (10) are expressed as

$$
\begin{aligned}
& x=\bar{a}+\bar{d} \leq-\sqrt{(\bar{a}-\bar{d})^{2}+4 \gamma^{2}}, \\
& x=\bar{b}+\bar{c} \leq-\sqrt{(\bar{b}-\bar{c})^{2}+4 \delta^{2}} .
\end{aligned}
$$

Without loss of generality we assume that $\lambda \geq \mu$, that is to say $a<b$. Since the right hand side of (12) is linear in $x$, in $(\bar{a}-\bar{d})$, and in $(\bar{b}-\bar{c})$, one has to saturate the constraints to maximize trace $(A R+B S)$. In other words, we must take

$$
\bar{a}-\bar{d}=\sqrt{x^{2}-4 \gamma^{2}}, \quad \text { and } \quad \bar{b}-\bar{c}=\sqrt{x^{2}-4 \delta^{2}} .
$$

Since $\delta \leq \gamma \leq 0$, this amounts to computing

$$
\max _{x \leq 2 \delta} f(x), \quad \text { with } f(x):=\frac{x}{2}+\frac{1}{4}(\lambda+\mu) \sqrt{x^{2}-4 \gamma^{2}}+\frac{1}{4}(\lambda-\mu) \sqrt{x^{2}-4 \delta^{2}} .
$$

We check that $f^{\prime}(x)$ is an increasing function of $x^{2}$, so that the maximum of $f(x)$ for $x \leq 2 \delta$ is attained at

$$
f^{\prime}(x)=0 \Longleftrightarrow x=-\frac{4 a b\left(1-\lambda^{2} \mu^{2}\right)}{\left(1-\lambda^{2}\right)\left(1-\mu^{2}\right)}, \quad \text { which implies } f(x)=-2 a b .
$$


We conclude from (12) that

$$
M K_{2}(R, S)^{2} \geq \operatorname{trace}(A R+B S) \geq a^{2}+b^{2}-2 a b=(a-b)^{2} .
$$

Together with (9), this implies that

$$
M K_{2}(R, S)^{2}=(a-b)^{2}=W_{2}\left(\frac{1}{2}\left(\delta_{-a}+\delta_{a}\right), \frac{1}{2}\left(\delta_{-b}+\delta_{b}\right)\right)^{2} .
$$

Therefore,

$$
C_{q}=C_{c}
$$

so that the classical and the quantum optimal transport costs are equal in this case.

\section{The Unequal Mass CASE}

In this section, we construct a family of density matrices $R$ and $S$ for which the quantum cost of optimal transport is smaller than the classical analogous cost.

With the same notations as in previous section, we set

$$
R:=\frac{1+\eta}{2}|a\rangle\left\langle a\left|+\frac{1-\eta}{2}\right|-a\right\rangle\left\langle-a\left|, \quad S:=\frac{1}{2}\right| a\right\rangle\left\langle a\left|+\frac{1}{2}\right|-a\right\rangle\langle-a|, \quad 0<\eta<1 .
$$

In other words, we consider the same situation as in the previous section with $a=b$, but with different masses for the quantum density matrix $R$.

In the orthonormal basis $\left\{\phi_{+}, \phi_{-}\right\}$, the density matrix $R$ takes the form

while $S$ is the same as before.

$$
R=\left(\begin{array}{cc}
\frac{1+\lambda}{2} & \frac{\eta}{2} \sqrt{1-\lambda^{2}} \\
\frac{\eta}{2} \sqrt{1-\lambda^{2}} & \frac{1-\lambda}{2}
\end{array}\right)
$$

We define the "quantized classical" coupling as

$$
Q_{c}:=\frac{1}{2}|a ; a\rangle\left\langle a ; a\left|+\frac{1-\eta}{2}\right|-a ;-a\right\rangle\left\langle-a ;-a\left|+\frac{\eta}{2}\right| a ;-a\right\rangle\langle a ;-a|,
$$

with the obvious notation

$$
\langle a ; b|:=\langle a|\otimes\langle b|; \quad| a ; b\rangle:=| a\rangle \otimes| b\rangle .
$$

Obviously $Q_{c} \geq 0$ by construction, and

$$
\operatorname{trace}_{2}\left(Q_{c}\right)=\frac{1}{2}|a\rangle\left\langle a\left|+\frac{\eta}{2}\right| a\right\rangle\left\langle a\left|+\frac{1-\eta}{2}\right|-a\right\rangle\langle-a|=R, \quad \text { while } \operatorname{trace}_{1}\left(Q_{c}\right)=S .
$$

Viewed as a matrix in the basis $\left\{\phi_{+} \otimes \psi_{+}, \phi_{+} \otimes \psi_{-}, \phi_{-} \otimes \psi_{+}, \phi_{-} \otimes \psi_{-}\right\}$,

$$
Q_{c}=\left(\begin{array}{cccc}
\frac{1}{4}(1+\lambda)^{2} & 0 & \frac{1}{4} \eta \sqrt{1-\lambda}(1+\lambda)^{\frac{3}{2}} & \frac{1}{4}(-1+\eta)\left(-1+\lambda^{2}\right) \\
0 & \frac{1}{4}\left(1-\lambda^{2}\right) & \frac{1}{4}(-1+\eta)\left(-1+\lambda^{2}\right) & \frac{1}{4} \eta(1-\lambda)^{\frac{3}{2}} \sqrt{1+\lambda} \\
\frac{1}{4} \eta \sqrt{1-\lambda}(1+\lambda)^{\frac{3}{2}} & \frac{1}{4}(-1+\eta)\left(-1+\lambda^{2}\right) & \frac{1}{4}\left(1-\lambda^{2}\right) & 0 \\
\frac{1}{4}(-1+\eta)\left(-1+\lambda^{2}\right) & \frac{1}{4} \eta(1-\lambda)^{\frac{3}{2}} \sqrt{1+\lambda} & 0 & \frac{1}{4}(-1+\lambda)^{2}
\end{array}\right) .
$$

With (7), we easily compute

$$
\operatorname{trace}\left(C Q_{c}\right)=2 \eta a^{2}=W_{2}\left(\frac{1+\eta}{2} \delta_{a}+\frac{1-\eta}{2} \delta_{-a}, \frac{1}{2} \delta_{a}+\frac{1}{2} \delta_{-a}\right)^{2} .
$$

Indeed, let us recall the classical optimal transport from $R$ to $S$ in this case: first, one "moves" the amount of mass $\frac{1}{2}$ from $a$ in $R$ to $a$ in $S$. The amount of mass $\frac{\eta}{2}$ remaining at $a$ in $R$ is transported to $-a$ in $S$, and the outstanding amount of mass $\frac{1-\eta}{2}$, located at $-a$ in $R$, is "transported" to $-a$ in $S$ (see Figure 2). 
For each $\epsilon>0$, set

$$
Q_{\epsilon}:=Q_{c}+\epsilon Q_{q},
$$

with

One easily checks that

$$
Q_{q}:=\left(\begin{array}{cccc}
1 & 0 & 0 & -1 \\
0 & -1 & 1 & 0 \\
0 & 1 & -1 & 0 \\
-1 & 0 & 0 & 1
\end{array}\right)
$$

$$
\operatorname{trace}_{1}\left(Q_{q}\right)=\operatorname{trace}_{2}\left(Q_{q}\right)=\operatorname{trace}\left(Q_{q}\right)=0,
$$

so that

$$
\text { trace }_{1}\left(Q_{\epsilon}\right)=S, \quad \text { and } \quad \operatorname{trace}_{2}\left(Q_{\epsilon}\right)=R, \quad \text { so that trace }\left(Q_{\epsilon}\right)=1 .
$$

The characteristic polynomial of $Q_{c}$ is found to be of the form

$$
\operatorname{det}\left(Q_{c}-t I\right)=t P_{3}(t)
$$

where $P_{3}$ is a cubic polynomial satisfying

$$
P_{3}(0)=-\frac{\eta}{8}(1-\eta)\left(1-\eta^{2}\right)<0 .
$$

Therefore the spectrum of $Q_{c}$ is $\left\{0, \lambda_{1}>0, \lambda_{2}>0, \lambda_{3}>0\right\}$ since $Q_{c}=Q_{c}^{*} \geq 0$. One can also check that

$$
\left.\operatorname{det}\left(Q_{\epsilon}-t I\right)\right|_{t=0}=\operatorname{det} Q_{\epsilon}=\epsilon \eta \lambda^{2}(1-\eta)\left(1-\lambda^{4}\right)+O\left(\epsilon^{2}\right)>0 \quad \text { for } 0<\epsilon \ll 1,
$$

together with

$$
\left.\frac{d}{d t} \operatorname{det}\left(Q_{c}-t I\right)\right|_{t=0}:=P_{3}(0)<0 .
$$

Hence there exists $D$ (independent of $\epsilon$ ) such that

$$
\left.\frac{d}{d t} \operatorname{det}\left(Q_{\epsilon}-t I\right)\right|_{t=0} \leq D<0 \quad \text { for } 0<\epsilon \ll 1 .
$$

Both (19) and (20) clearly imply that $\operatorname{det}\left(Q_{\epsilon}-t I\right)$ has a positive zero that is $\epsilon$-close to 0 , and three other roots which are $\epsilon$-close to $\lambda_{1}, \lambda_{2}$ and $\lambda_{3}>0$ respectively. Therefore, $Q_{\epsilon}=Q_{\epsilon}^{*}>0$ for $0<\epsilon \ll 1$, and (18) implies that $Q_{\epsilon}$ is a coupling of $R$ and $S$.

Another elementary computation shows that

so that

$$
\operatorname{trace}\left(C Q_{q}\right)=-\frac{8 a^{2} \lambda^{2}}{1-\lambda^{2}}
$$

$$
\begin{aligned}
M K_{2}(R, S)^{2} & \leq \operatorname{trace}\left(C Q_{\epsilon}\right)=\operatorname{trace}\left(C Q_{c}\right)-\epsilon \frac{8 a^{2} \lambda^{2}}{1-\lambda^{2}} \\
& <W_{2}\left(\frac{1+\eta}{2} \delta_{a}+\frac{1-\eta}{2} \delta_{-a}, \frac{1}{2} \delta_{a}+\frac{1}{2} \delta_{-a}\right)^{2},
\end{aligned}
$$

for each $\epsilon$ satisfying $0<\epsilon \ll 1$, according to formula (16). In other words,

$$
C_{q}<C_{c}
$$

the quantum cost is (strictly) below the classical cost. 


\section{Concluding REMARKS ON QUANTUM Optimal TRANSPORT}

The result of Section 2 shows that, in the equal mass case, an optimal coupling is given by the following matrix in the basis $\left\{\phi_{+} \otimes \psi_{+}, \phi_{+} \otimes \psi_{-}, \phi_{-} \otimes \psi_{+}, \phi_{-} \otimes \psi_{-}\right\}$:

$$
Q=\frac{1}{4}\left(\begin{array}{cccc}
1+\lambda \mu+\lambda+\mu & 0 & 0 & \sqrt{(1+\lambda \mu)^{2}-(\lambda+\mu)^{2}} \\
0 & \frac{1-\lambda \mu+\lambda-\mu}{(1-\lambda \mu)^{2}-(\lambda-\mu)^{2}} & \sqrt{(1-\lambda)^{2}-(\lambda-\mu)^{2}} & 0 \\
1-\lambda \mu-\lambda+\mu & 0 \\
\sqrt{(1+\lambda \mu)^{2}-(\lambda+\mu)^{2}} & 0 & 0 & 1+\lambda \mu-\lambda-\mu
\end{array}\right) .
$$

Using (6) and with the same notation as in (14), the optimal coupling $Q$ can be put in the form

$$
Q=\frac{1}{2}(|a ; b\rangle\langle a ; b|+|-a ;-b\rangle\langle-a ;-b|) .
$$

In other words, $Q$ is the Töplitz operator of symbol

$$
\Pi\left(q, p ; q^{\prime}, p^{\prime}\right)=\frac{1}{2} \delta_{(-a, 0)}(q, p) \delta_{(-b, 0)}\left(q^{\prime}, p^{\prime}\right)+\frac{1}{2} \delta_{(a, 0)}(q, p) \delta_{(b, 0)}\left(q^{\prime}, p^{\prime}\right) .
$$

Likewise, we recall that $R$ is the Töplitz operator of symbol

$$
\mu(q, p)=\frac{1}{2}\left(\delta_{(-a, 0)}(q, p)+\delta_{(a, 0)}(q, p)\right)
$$

while $S$ is the Töplitz operator of symbol

$$
\nu(q, p)=\frac{1}{2}\left(\delta_{(-b, 0)}(q, p)+\delta_{(b, 0)}(q, p)\right) .
$$

Therefore,

$$
\begin{aligned}
\Pi\left(q, p ; q^{\prime}, p^{\prime}\right) & =\frac{1}{2}\left(\left(\delta_{(-a, 0)}(q, p)+\delta_{(a, 0)}(q, p)\right) \delta\left(\left(q^{\prime}, p^{\prime}\right)-\Phi(q, p)\right)\right) \\
& =\mu(q, p) \delta\left(\left(q^{\prime}, p^{\prime}\right)-\Phi(q, p)\right),
\end{aligned}
$$

where $\Phi$ is any map satisfying $\Phi(a, 0)=(b, 0)$ and $\Phi(-a, 0)=(-b, 0)$.

The second equality in (23) says the following: in the equal mass case, in agreement with (3), an optimal quantum coupling $Q$ is the Töplitz operator of symbol the classical optimal coupling associated to the optimal transport map

$$
((-a, 0),(a, 0)) \mapsto((-b, 0),(b, 0)) .
$$

In the unequal mass case treated in Section 3, the coupling $Q_{c}$ defined by (14) is also a Töplitz operator, with symbol

$$
\begin{aligned}
\Pi_{c}\left(q, p ; q^{\prime}, p^{\prime}\right) & =\frac{1}{2} \delta_{(a, 0)}(q, p) \delta_{(a, 0)}\left(q^{\prime}, p^{\prime}\right) \\
& +\frac{1-\eta}{2} \delta_{(-a, 0)}(q, p) \delta_{(-a, 0)}\left(q^{\prime}, p^{\prime}\right)+\frac{\eta}{2} \delta_{(a, 0)}(q, p) \delta_{(-a, 0)}\left(q^{\prime}, p^{\prime}\right) .
\end{aligned}
$$

This expression is easily interpreted as the optimal coupling associated to the "transport" introduced in Section 1, Figure 2, exactly as in the equal mass case. But, as explained in the previous section, $Q_{c}$ cannot be an optimal coupling, since the coupling $Q_{\epsilon}$ defined by (17) leads to a strictly lower quantum cost.

We did not compute any optimal coupling in this situation. Observe however that, thanks to (15) and (6) specialized to $a=b$ (so that $\lambda=\mu$ ), one can expand $Q_{q}$ in the form

$$
Q_{q}=\sum_{i, j, k, l= \pm 1} q_{i, j, k, l}|i a ; j a\rangle\langle k a ; l a|
$$


The contribution of the "diagonal" terms $q_{i, j, i, j}$ defines a Töplitz operator, unlike the off-diagonal terms such as $q_{1,1,-1,1}=\frac{-4 \lambda}{\left(1-\lambda^{2}\right)^{2}} \neq 0$ for instance.

In general, when $R$ and $S$ are Töplitz operators of symbols $\mu$ and $\nu$ satisfying $M K_{2}(R, S)<W_{2}(\mu, \nu)$, no optimal coupling $Q_{o p}$ of $R$ and $S$ can be a Töplitz operator: if such was the case, the Töplitz symbol of $Q_{o p}$ would be a coupling of $\mu$ and $\nu$ with classical transport cost $M K_{2}(R, S)<W_{2}(\mu, \nu)$, which is impossible. The presence of nonclassical off-diagonal terms in $Q_{o p}$, such as $q_{1,1,-1,1}=\frac{-4 \lambda}{\left(1-\lambda^{2}\right)^{2}} \neq 0$ in the example discussed above, are precisely the reason why quantum optimal transport can be cheaper in this case than classical optimal transport.

Finally, observe that both $W_{2}\left(\frac{1+\eta}{2} \delta_{a}+\frac{1-\eta}{2} \delta_{-a}, \frac{1}{2} \delta_{a}+\frac{1}{2} \delta_{-a}\right)^{2}-\operatorname{trace}\left(C Q_{\epsilon}\right)$ and $q_{1,1,-1,1}$ are exponentially small as $\hbar \rightarrow 0$, but of course are not small for $\hbar=1$.

Acknowledgements. This work has been partially carried out thanks to the support of the LIA AMU-CNRS-ECM-INdAM Laboratoire Ypatie des Sciences Mathématiques (LYSM).

\section{REFERENCES}

[1] A.G. Athanassoulis, N.J. Mauser, T. Paul: Coarse-scale representations and smoothed Wigner transforms, J. Math. Pures Appl. 91 (2009), 296-338.

[2] H. Brezis: Remarks on the Monge-Kantorovich problem in the discrete setting, C. R. Acad. Sci. Paris, Sér. I 356 (2018), 207-213.

[3] E. Caglioti, F. Golse, T. Paul: Towards optimal transport for quantum densities, preprint hal-01963667, (2018).

[4] E.A. Carlen, J. Maas: An Analog of the 2-Wasserstein Metric in Non-Commutative Probability Under Which the Fermionic Fokker-Planck Equation is Gradient Flow for the Entropy, Commun. Math. Phys., 331 (2014), 887-926.

[5] E.A. Carlen, J. Maas: Non-commutative calculus, optimal transport and functional inequalities in dissipative quantum systems, J. Stat. Phys. 178 (2020), 319-378.

[6] G. De Palma, D. Trevisan: Quantum optimal transport with quantum channels, preprint arXiv:1911.00803 [math-ph]

[7] F. Golse, C. Mouhot, T. Paul: On the Mean-Field and Classical Limits of Quantum Mechanics, Commun. Math. Phys. 343 (2016), 165-205.

[8] F. Golse, T. Paul: The Schrödinger Equation in the Mean-Field and Semiclassical Regime, Arch. Rational Mech. Anal. 223 (2017), 57-94.

[9] F. Golse, T. Paul: Wave Packets and the Quadratic Monge-Kantorovich Distance in Quantum Mechanics, Comptes Rendus Mathématique 356 (2018), 177-197.

[10] K. Ikeda: Foundation of Quantum Optimal Transport and Applications, preprint arXiv:1906.09817 [quant-ph].

[11] M. Mézard, G. Parisi: The Euclidean Matching Problem, J. Phys. France 49 (1988), 2019-2025. 
[12] C. Rouze, N. Datta: Relating relative entropy, optimal transport and Fisher information: a quantum HWI inequality, preprint arXiv:1709.07437 [quant-ph], to appear in Ann. Henri Poincaré (2020) doi:10.1007/s00023-020-00891-8

[13] G. Sicuro: "The Euclidean Matching Problem", Springer-Verlag, Berlin, Heidelberg, 2017.

[14] C. Villani: "Topics in Optimal Transportation", Amer. Math. Soc., Providence (RI), 2003.

[15] C. Villani: "Optimal Transport. Old and New", Springer-Verlag, Berlin, Heidelberg, 2009.

[16] K. Zyckowski, W. Slomczynski Monge Distance between Quantum States, J. Phys. A 31 (1998), 9095-9104.

(E.C.) Sapienza Università di Roma, Dipartimento di Matematica, Piazzale Aldo Moro 5, 00185 Roma

E-mail address: caglioti@mat.uniroma1.it

(F.G.) CMls, Ecole polytechnique, 91128 Palaiseau Cedex, France

E-mail address: francois.golse@polytechnique.edu

(T.P.) CMls, CNRS, Ecole polytechnique, 91128 Palaiseau Cedex, France

E-mail address: paul@ljll.math.upmc.fr 